\title{
Operative versus non-operative treatment for closed, displaced, intra-articular fractures of the calcaneus: randomised controlled trial
}

\author{
(c) $\underset{\text { (1) () }}{\mathrm{gy}}$ OPEN ACCESS
}

\begin{abstract}
Damian Griffin professor of trauma and orthopaedic surgery, Nick Parsons senior research fellow in medical statistics, Ewart Shaw associate professor in statistics, Yuri Kulikov clinical research fellow, Charles Hutchinson professor of clinical imaging, Margaret Thorogood professor of epidemiology, Sarah E Lamb professor of rehabilitation, for the UK Heel Fracture Trial (UK HeFT) investigators
\end{abstract}

Warwick Medical School and Department of Statistics, University of Warwick, and University Hospital of Coventry and Warwickshire NHS Trust, Coventry, UK

\begin{abstract}
Objective To investigate whether surgery by open reduction and internal fixation provides benefit compared with non-operative treatment for displaced, intra-articular calcaneal fractures.

Design Pragmatic, multicentre, two arm, parallel group, assessor blinded randomised controlled trial (UK Heel Fracture Trial).

Setting 22 tertiary referral hospitals, United Kingdom

Participants 151 patients with acute displaced intra-articular calcaneal fractures randomly allocated to operative $(n=73)$ or non-operative $(n=78)$ treatment.
\end{abstract}

Main outcome measures The primary outcome measure was patient reported Kerr-Atkins score for pain and function (scale 0-100, 100 being the best possible score) at two years after injury. Secondary outcomes were complications; hindfoot pain and function (American Orthopaedic Foot and Ankle Society score); general health (SF-36); quality of life (EQ-5D); clinical examination; walking speed; and gait symmetry. Analysis was by intention to treat.

Results 95\% follow-up was achieved for the primary outcome (69 in operative group and 74 in non-operative group), and a complete set of secondary outcomes were available for $75 \%$ of participants. There was no significant difference in the primary outcome (mean Kerr-Atkins score 69.8 in operative group $v 65.7$ in non-operative group; adjusted $95 \%$ confidence interval of difference -7.1 to 7.0 ) or in any of the secondary outcomes between treatment groups. Complications and reoperations were more common in those who received operative care (estimated odds ratio $7.5,95 \%$ confidence interval 2.0 to 41.8 ).

Conclusions Operative treatment compared with non-operative care showed no symptomatic or functional advantage after two years in patients with typical displaced intra-articular fractures of the calcaneus, and the risk of complications was higher after surgery. Based on these findings, operative treatment by open reduction and internal fixation is not recommended for these fractures.

Trial registration Current Controlled Trials ISRCTN37188541.

\section{Introduction}

Fractures of the calcaneus, or heel bone, make up about $2 \%$ of all fractures and are the commonest fracture of the tarsal bones. ${ }^{1}$ Some calcaneal fractures are minor injuries, but many are severe, high energy fractures. These more serious injuries usually occur after a fall from a height, often from scaffolding or a ladder, or as a result of a road traffic incident. In 2010, 2721 people in England, ${ }^{2}$ and 17274 in the United States, ${ }^{3}$ were admitted to hospital with these serious injuries, typically with an inpatient stay of more than a week. The incidence is even higher in developing countries. ${ }^{4}$

These calcaneal injuries are destructive, with fracture and displacement of the whole bone and its joint surfaces; the subtalar joint in particular may be severely disrupted. With conservative treatment the fracture fragments usually heal together, but the calcaneus remains deformed, the joint surfaces are incongruous, and the alignment of the leg through the ankle to the heel is lost. Severe, painful osteoarthritis of the subtalar joint often follows. Recovery is prolonged, typically taking two years. Even then, most patients have a painful, stiff, deformed foot, and are unable to wear a normal shoe; walking is painful and many need the assistance of a walking stick. ${ }^{6}$ These poor outcomes are especially problematic for the typical patient who is a labourer or outdoor worker, as they are unable to resume their occupation. This affect on working life was recognised as early as 1916: "Ordinarily speaking, the man who breaks his heel bone is done, so far as his industrial future is concerned."7 
In developing countries especially, this loss of economic potential has a negative effect on patients and their families. In developed countries, healthcare and societal costs are high because of the long hospital stay, extended treatment, delayed or non-return to work, and long term disability benefits. ${ }^{8}$

Conservative, non-operative care includes elevation, application of ice, early mobilisation, and the use of a splint. ${ }^{6}$ Orthopaedic surgeons have aimed to treat severe calcaneal fractures to accelerate recovery and reduce pain and deformity. In the 1950s, operative treatment with a percutaneous "spike" became popular and was widely performed. ${ }^{9}$ In the 1960 s, enthusiasm waned because of reported difficulties of the technique..$^{10}$ In the 1980s, limited exposure of the subtalar joint and fixation with wires was attempted, but a randomised controlled trial showed no benefit from surgery. ${ }^{11}$ In the 1990s, computerised tomography allowed a better understanding of fracture patterns, and new surgical approaches were developed that allowed surgeons to realign the bone fragments, fix them with plates and screws, and restore the subtalar joint. ${ }^{12}$ Observational studies of these treatments ${ }^{13}$ reported low complication rates and better clinical outcomes than had been observed in historical non-operative series, and this new surgical treatment of severe calcaneal fractures rapidly spread in Europe, the United States, Canada, and Australia. It is now being promoted in developing countries. $^{14}$

Four independent systematic reviews, ${ }^{15-18}$ including a recent Cochrane review, have examined the controlled evidence for the effectiveness of this surgery. All four reviews remarked on the paucity of evidence and the poor quality of studies to date. One suggested that surgery might lead to better functional recovery than conservative care, but all noted the risk of complications after surgery, including infection and the need for reoperation. All concluded that the available evidence is insufficient to choose the best management strategy for these fractures.

The current situation is one of uncertainty. Some orthopaedic surgeons are enthusiastic about this surgery for calcaneal fractures, and recommend it to patients. Others consider the operations to be complex, expensive, risky, ${ }^{19}$ and without proved benefit, and so recommend non-operative care. The dilemma of how best to treat such patients is a familiar one in hospitals worldwide; whether a patient who sustains this fracture in the United Kingdom today undergoes surgery depends to an extent on the hospital and the surgeon.

We performed a large, pragmatic, randomised controlled trial in the UK National Health Service to assess whether operative care leads to better outcomes than non-operative care in patients with typical, closed, displaced intra-articular calcaneal fractures over two years after injury.

\section{Methods}

\section{Study design and participants}

We carried out a pragmatic, multicentre, two arm, parallel group, assessor blinded randomised controlled trial with 1:1 treatment allocation. Patients were eligible if they were aged 18 years or more, and able to give informed consent, with a recent (less than three weeks) closed, intra-articular, displaced (subtalar joint posterior facet displacement of at least $2 \mathrm{~mm}$ ) calcaneal fracture. Exclusions were gross deformity of the hindfoot (which we called fibula impingement and defined as: such severe calcaneal varus that after healing patients would walk on the tip of the fibula; or such severe calcaneal valgus that the tip of the fibula was embedded in the lateral wall of the calcaneus), other serious leg injuries sufficient to affect outcome at two years, not fit for surgery, peripheral vascular disease, or inability to adhere to the trial procedures. We included patients with bilateral fractures unless one or both of the fractures met the exclusion criteria.

\section{Recruitment and randomisation of participants}

We recruited participants from 22 UK hospitals in 2007-09. All were regional referral centres for calcaneal fractures, and the surgeons were all recognised as specialists in the treatment of these injuries. The calcaneal fractures seen in the emergency department or referred to orthopaedic surgeons at each centre during the recruitment period were identified prospectively. To ensure identification of all eligible patients, we audited attendance at the emergency departments and the radiology records.

Surgeons in each centre identified potential participants, assessed eligibility, and graded each fracture into one of four types according to Sanders classification: 1=non-displaced fractures (displacement $<2 \mathrm{~mm}$ ); 2=two-part or split fractures of the subtalar joint; $3=$ three-part or split depression fractures; and 4=four-part or highly comminuted articular fractures. ${ }^{20}$ Research associates approached eligible patients to explain the trial, supported by a DVD presentation. Patients who consented to participate were randomised 1:1 to receive operative or non-operative care, using a mimimisation algorithm for centre, smoking status, diabetes, bilaterality, and Sanders classification. Randomisation was by participant, so those with bilateral fractures were allocated the same treatment on both sides. A secure telephone randomisation service ensured allocation concealment. We collected baseline data before randomisation. All participants provided written informed consent.

\section{Interventions}

Initial treatment for all participants was bed rest, analgesia, elevation of the foot, and application of ice.

Operative treatment was open reduction and internal fixation within three weeks of injury, performed through an extensile lateral approach, with interfragmentary screws and application of a neutralisation plate or plates to the lateral wall of the calcaneus. ${ }^{12}$ This surgical technique was chosen because it is considered to be the standard of care in the United States, United Kingdom, and most centres around the world. ${ }^{21}$ Postoperative care included application of a splint, six weeks non-weight bearing followed by six weeks partial weight bearing, with early active mobilisation of the ankle and subtalar joints, managed by a standardised physiotherapy rehabilitation regimen. Postoperative computed tomography was performed within three months of surgery; an independent radiologist $(\mathrm{CH})$ classified the technical outcome of surgery by accuracy of reduction.

Non-operative treatment began with gentle mobilisation of the ankle and subtalar joints as pain allowed, and the fitting of a removable splint. Participants were mobilised and non-weight bearing (on the affected side) for six weeks, followed by six weeks partial weight bearing. They were managed by the same standardised physiotherapy rehabilitation regimen as the operative treatment arm. Compliance with the physiotherapy protocol was assessed by a proforma completed by the treating physiotherapist, and by questions in the patient assessments at six weeks and six months. 


\section{Outcome measurement}

The primary outcome instrument was the Kerr-Atkins calcaneal fracture score. ${ }^{22}$ This is a validated patient reported instrument quantifying pain and function after calcaneal fracture. One hundred points represent normal pain and function. Scores in the range 80-100 indicate either slight pain or minor restrictions to walking ability, such as occasional use of a walking stick, and scores in the range 60-80 indicate moderate pain, restricted walking, and more than occasional use of a walking stick. We measured the primary outcome at two years after injury when at least two thirds of the patients would be expected to have reached maximal recovery. ${ }^{23}$ Quality of life and general health status were measured using the EuroQol EQ-5D ${ }^{24}$ and SF-36. ${ }^{25}$ We used the American Orthopaedic Foot and Ankle Society score to assess global hindfoot function ${ }^{26}$; the instrument is not specific to the calcaneus, but it has been widely used in studies of foot and ankle surgery. Patient reported outcomes were gathered before randomisation, and by postal questionnaires after 6,12 , and 18 months. Complications, additional surgery, and return to work were recorded at a six week follow-up consultation, and at $6,12,18$, and 24 months.

At two years, participants completed a final set of Kerr-Atkins, EQ-5D, SF-36, and American Orthopaedic Foot and Ankle Society score questionnaires, and a single physiotherapist, who was unaware of treatment allocation, performed a clinical assessment. Medial to lateral heel width was measured with a caliper at the widest point of the heel distal to the malleoli. Hindfoot range of movement was measured with a goniometer with the participant sitting. ${ }^{27}$ Walking speed was measured over a $6 \mathrm{~m}$ track, with $1 \mathrm{~m}$ at either end for acceleration and deceleration, during five trials. Gait was assessed with an in-shoe pedobarographic system (F-Scan; Tekscan, Boston, MA). We recorded and averaged each step for both feet. For the injured and non-injured feet (bilateral fractures were excluded) we calculated contact duration; peak pressures for midfoot, first metatarsal head, and fifth metatarsal head; and force-time integral (impulse). During this examination, participants wore thin socks to obscure surgical scars and maintain blinding of the physiotherapist.

\section{Statistical analysis}

Through discussion with surgeon collaborators during a pilot study in 24 patients we agreed a minimum clinically important difference of 15 points in the Kerr-Atkins score. These patients met the same inclusion criteria as for this trial, and were operated on by two surgeons. Fifteen points corresponds to the difference between, for instance, moderate pain and no pain, or between moderate restriction and an unrestricted ability to walk..$^{22}$ Whereas a smaller 10 point difference in Kerr-Atkins score would analogously correspond to the difference between slight pain and no pain, or between minimal restriction and an unrestricted ability to walk. The pilot study gave an estimate for the standard deviation of 20 points, similar to that of other studies (14 and 15-20 points in two case series ${ }^{1328}$ ). We designed the study to detect a treatment effect of 10 points with $80 \%$ power at the $5 \%$ significance level, which required data from 126 participants. Allowing for approximately $20 \%$ loss to follow-up, we sought to recruit 150 participants.

For the main analysis we assessed differences in the Kerr-Atkins score at two years after injury between treatment groups on an intention to treat basis. We summarised primary and secondary outcomes using means (standard deviations) and treatment effects as the difference in means (with 95\% confidence intervals). Estimates of treatment effects were obtained using mixed effects regression models with adjustment for the fixed effects of baseline (pre-injury) scores, age, sex, smoking status, diabetes, employment status, Sanders classification, and compensation status, ${ }^{29}$ and for the random effect of recruiting centre. We used conventional linear models for all outcomes based on assumed approximate normality, other than return to work where we used a logistic regression model. For the primary outcome, we regarded $\mathrm{P}$ values of less than 0.05 to be significant; however, to adjust for multiple testing, for all secondary outcomes we regarded $P$ values of less than 0.01 to be significant. To investigate the moderating effects of Sanders classification on treatment we planned subgroup analyses for the primary outcome measure, and we used a formal interaction test to assess these effects in the regression model. ${ }^{30} \mathrm{We}$ used $\chi^{2}$ tests or Fisher's exact tests (for infrequent events) to compare complications between groups as well as sex and age profiles of participants who were recruited, ineligible, or refused to take part in the trial. For other outcome data, we used $t$ tests and Wilcoxon rank sum tests to compare the treatment groups under assumed approximate normality and non-normality of outcome distributions. For participants with bilateral fractures, we collected outcome measures for the more severely injured side, with analysis for that fracture. For data on gait, we used a symmetry index to describe the difference between injured and non-injured feet. This was calculated as the difference in a metric divided by the mean of that metric for both sides. ${ }^{31} \mathrm{~A}$ value of $0 \%$ indicated no imbalance (complete symmetry) and a negative value indicated that the metric was smaller for the injured foot than for the non-injured (control) foot.

\section{Results}

Overall, 2006 patients presented to the collaborating centres with calcaneal fractures. Of these, $502 \mathrm{had}$ severe fractures that met the eligibility criteria (fig $1 \Downarrow$ ). An audit of all centres identified only three further patients with displaced, intra-articular fractures who might have been eligible for the trial. Of the 502 eligible patients, 151 consented to participate and were randomised to the operative $(n=73)$ and non-operative $(n=78)$ groups, with a median of 5 (interquartile range 4-8) participants per centre. The mean age of participants was 46.5 years (range 18-80), and 24 (16\%) were women. No significant differences were found between treatment groups in sex, age, body mass index, smoking, prevalence of diabetes, or baseline scores (table $1 \Downarrow$ ). Follow-up for the primary outcome after two years was $95 \%$ in each group. All of the secondary outcome measures at all time points were available for $75 \%$ of the patients.

In total, 351 patients were eligible but declined to participate; there were no significant differences between those who consented to participate and those who did not. The commonest reason for patients declining $(n=290,83 \%)$ was a preference for operative or non-operative care, with preferences equally balanced (144 and 146, respectively).

Operations were performed by 27 surgeons across the 22 centres. No centre had more than two surgeons; the median number of operations per surgeon was two with a maximum of six. Postoperative computed tomography was performed on 51 (72\%) of those who received operative treatment; the remaining 20 participants did not attend their scan appointments. Accuracy of the operative reduction was rated on the scans as no more than a $2 \mathrm{~mm}$ step in the articular surface $(\mathrm{n}=40 ; 78 \%, 95 \%$ confidence interval $64 \%$ to $88 \%$ ), a residual step of more than $2 \mathrm{~mm}(\mathrm{n}=7)$, and a residual step greater than $2 \mathrm{~mm}$ and poor alignment of the calcaneal body $(n=4)$. 
Most of the trial participants attended for outpatient physiotherapy, $65 / 73$ (89\%, $95 \%$ confidence interval $79 \%$ to $95 \%)$ in the operative group and $66 / 78(85 \%, 74 \%$ to $91 \%)$ in the non-operative group $(\mathrm{P}=0.27)$. Twelve did not attend scheduled outpatient physiotherapy sessions (three operative and eight non-operative); in eight their use of physiotherapy was not known (three left the United Kingdom, two withdrew from the trial, and three were lost to follow-up). The median number of sessions attended was eight (range 1-30) in the operative group and six (range 1-30) in the non-operative group, with no evidence $(\mathrm{P}=0.22)$ that attendance at physiotherapy sessions differed between the intervention groups.

Kerr-Atkins scores improved for 18 months after injury and were then stable to the trial endpoint at two years for the full population as well as for subgroups with type 2 fractures and with type 3 and 4 fractures according to Sanders classification (fig $2 \Downarrow$ ). At two years, Kerr-Atkins scores were in the range 60-80 and still considerably lower than before injury, indicating moderate pain and changes to normal walking. This was confirmed by the SF-36 physical component scores, which were approximately 40 at two years, a level associated with longstanding physical illness. ${ }^{32}$

On intention to treat analysis (table $2 \Downarrow$ ), the Kerr-Atkins scores were similar between operative (69.8) and non-operative (65.7) groups after two years, with an unadjusted effect size of 4.1 points ( $95 \%$ confidence interval -3.4 to $11.5, \mathrm{P}=0.28$ ). No treatment effect was observed after adjustment for potential confounders (difference in Kerr-Atkins score -0.03 , 95\% confidence interval -7.08 to 7.02 ). Although the trial was not formally powered to assess the effects of fracture severity on outcome, table 2 shows estimated treatment effects for subgroups according to Sanders classification; a formal interaction test in the regression analysis showed no evidence $(\mathrm{P}=0.697)$ that the effect of surgery was affected by fracture severity. There was no evidence for treatment effects for general health (SF-36 physical or mental component scores), quality of life (EQ5-D), or hindfoot pain and function (American Orthopaedic Foot and Ankle Society score), table 2). Neither were there treatment effects of surgery for heel width, range of motion, walking speed, or any other metrics of gait symmetry. Range of motion and gait symmetry data are expressed as percentage changes compared with the non-injured foot in table 2 , with raw data in figures $3 \Downarrow$ and $4 \Downarrow$.

Significantly more patients experienced complications and reoperations (table $3 \Downarrow$ ) in the operative group $(17 / 73 ; 23 \%)$ than in the non-operative group $(3 / 78 ; 4 \%)$; estimated odds ratio 7.5 (95\% confidence interval 2.0 to 41.8 , Fisher's exact test $\mathrm{P}$ $<0.001)$. The most common complication was surgical site infection. All of these infections occurred in the operative group, usually within six weeks, and 5/14 required remedial surgery. Three more patients had reoperations to remove painful, prominent screws and plates. By two years, three subtalar fusions for painful arthritis had been performed in the non-operative group, but none in the operative group.

Since eight patients had crossed over between treatment allocation groups, all the above intention to treat analyses were repeated on an as treated basis. This also provided no evidence for significant treatment effects-for example, the main result of the difference in the mean Kerr-Atkins score between those who had surgery and those who did not was -1.08 points $(95 \%$ confidence interval -8.10 to 5.95 ).

\section{Discussion}

Surgical treatment of typical, closed, displaced calcaneal fractures does not improve outcome when compared with non-operative treatment, and leads to an increase in serious complications. We found no difference in patient reported outcome at two years between those treated by open reduction and internal fixation and those treated non-operatively. There was also no treatment effect on any of the secondary outcome measures at any time point, including subjective and objective measures, which reinforces our main result. We had anticipated benefit in at least some of these measures, but there were no differences in patient reported general health, quality of life, or ability to return to work. Ranges of movement of the injured hind foot were no different between treatment groups, and even heel width (a commonly suggested reason for surgery, to facilitate shoe fitting) was the same. Walking speed and five different metrics of gait also showed no difference at two years after injury.

Complication rates were much higher in the operatively treated group, with a $19 \%$ infection rate and an $11 \%$ requirement for secondary surgery to remove infected or painful screws and plates. These are serious and expensive complications, which can be avoided by choosing non-operative care.

Participants in this study recovered slowly, regardless of their treatment, reaching a plateau of improvement at about 18 months. After two years, most were still adversely affected by their injury. About $85 \%$ had returned to work, although most changed to less physically demanding work, with no difference between groups.

Longer term follow-up may reveal differences in consequences between treatment groups, such as the progression of arthritis, the need for late subtalar fusion surgery, or better results of late fusion when primary open reduction and internal fixation were performed. Our results support this possibility, with three fusions performed in the non-operative group and none in the operative group. However, we believe that it is too early to draw such a conclusion, and we plan to report comparisons after five years of follow-up.

\section{Strengths and limitations of this study}

Our study was a pragmatic trial involving 22 hospitals and 27 specialist surgeons and so can be relied on to give a realistic assessment of effectiveness of surgery in the real world; no individual surgeon undertook more than six procedures, and the median number of procedures for each surgeon was two. The best recruiting centre recruited $13 \%$ of the study population and the best five centres together recruited just over $50 \%$ of the total; this represented a typical spread of recruitment numbers, which reflected true variability in performance between centres and the fact that centres came into the study sequentially over a considerable period, and so the number of months each centre had been recruiting into the study differed markedly between centres. There was no evidence to suggest that study participants differed in severity of fractures between recruitment centres. Although a few patients had less than perfect fracture reductions, this illustrates the difference between measurement of effectiveness and efficacy and the value of a pragmatic trial. Participants were drawn from a large cohort of almost all patients with calcaneal fractures presenting to emergency departments during the trial. Most patients who were excluded had fractures that did not require surgery (extra-articular or undisplaced), whereas some had open or severely displaced fractures that absolutely required surgery. Patients who were 
included represent those for whom there is most uncertainty around the best treatment choice.

All of the surgeons who took part were considered to be experts by their peers. They are the surgeons to whom patients with calcaneal fractures are referred from across the United Kingdom. It is possible that a surgeon with an extremely specialised practice, and with a high volume of this type of surgery, could achieve a better outcome. We have no evidence to support this, and such a trial could not be performed in the United Kingdom. This study provides a pragmatic result for the UK National Health Service, which reflects current surgical practice, case mix, and organisation of services in the United Kingdom.

Recruitment rate was $30 \%$ among eligible patients, which is at least as high as has been achieved in other surgical trials of operative compared with non-operative care. We are confident that our result is generalisable because those who agreed and those who declined to participate were similar in all factors that we found to be associated with variation in outcome. The follow-up rate was $95 \%$ for the primary outcome measure, giving us confidence in our estimate of effect. There was no significant change in this estimate between an intention to treat analysis and a per protocol analysis, indicating that the small crossover rate $(5 \%)$ did not influence our results.

The observed standard deviation of the primary outcome was 24 points compared with the 20 points we used for the power calculation. However, the confidence interval for the primary outcome excludes a treatment effect of 10 points, which we designed the trial to detect. The sample size was large enough to detect this magnitude of treatment effect if it exists. We cannot exclude a smaller effect, but our data do not suggest that such a benefit exists. Even if it did, a larger trial may not be feasible (a similar Dutch study closed early in 2012 after problems with recruitment ${ }^{18}$ ), and it seems unlikely that such a small beneficial treatment effect would warrant the increased risk of complications that we observed.

We studied patients with closed, displaced fractures. Forty five $(0.2 \%)$ open fractures and $57(0.3 \%)$ grossly displaced fractures showing "fibula impingement" were excluded from the study. Most of these were treated operatively. Some were treated by external fixation, some by primary fusion, some by amputation, and some by open reduction and internal fixation. Before the study began, we identified these as types of fracture that were likely to need operative treatment, and our results do not apply to these groups.

We have studied the effectiveness of open reduction and internal fixation for calcaneal fractures. Other surgical techniques have recently been proposed, including percutaneous fixation. ${ }^{21}$ These techniques may be safer and more effective, as their proponents claim, but we believe that the results of this study should make us cautious about adopting them outside of a clinical trial.

\section{Comparison with other studies}

A recent Cochrane review concluded that there was insufficient high quality evidence to establish whether surgical or conservative treatment was better for adults with displaced intra-articular calcaneal fractures, and called for an adequately powered, multicentre controlled trial. ${ }^{18}$

Three trials have compared open reduction and internal fixation with non-operative care in patients with displaced intra-articular calcaneal fractures, and these showed an advantage with surgery. Two small trials were not randomised ${ }^{33}{ }^{34}$ and so are at high risk of selection bias. One randomised trial ${ }^{35}$ reported benefits from surgery assessed by a functional questionnaire $(87 / 100 v 55 / 100$; $\mathrm{P}<0.001$ ) and a qualitative grading of outcome. However, this study included only 30 patients and had a $13 \%$ loss to follow-up at a mean of 15 months. An independent clinical examination also found benefit of surgery, but only $37 \%$ of participants were assessed in this way, raising a high probability of attrition bias. Two further randomised trials have been reported comparing open reduction and internal fixation with non-operative care in patients with displaced intra-articular calcaneal fractures. The first was a Canadian trial ${ }^{29}$ that included 424 patients. Unusually, it used a pre-randomisation strategy ${ }^{36}$ in which potential participants were randomised and then asked to consent to the allocated treatment. The trial used two principal outcome measures: short form 36 (SF-36) and a visual analogue score for pain. Outcome was assessed in $73 \%$ of participants. The authors concluded that there was no overall benefit from surgery, but that on subgroup analysis, some groups of patients did better with than without surgery. Although statistically significant, these benefits may not have been clinically significant, or at least were not defined as such pre-hoc. One surgeon performed the majority of operations (73\%), limiting the generalisability of the results. The large volume of operations done by one surgeon may have contributed to a difference between this trial and ours: the Canadian trial showed a benefit of surgery for type 2 fractures according to the Sanders classification; in our more generalisable study we showed no such benefit. We used the Canadian trial to plan our study, dealing with the criticisms that have been made of it. In particular, we avoided selection bias by randomising all eligible consenting patients and allowing no post-randomisation exclusions, ensured generalisability by including many surgeons in many centres, avoided attrition bias by achieving a much higher rate of follow-up (95\%), used a disease specific outcome instrument, and prespecified the subgroup analyses.

The second trial, ${ }^{37}$ from five centres in Sweden, was published since we began our study and since the recent Cochrane systematic review. Eighty two patients were randomised to open reduction and internal fixation or non-operative care. Outcome measures were similar to those in the Canadian trial, and follow-up rates were $93 \%$ at one year and $71 \%$ at $8-12$ years. At one year, there were no differences between groups, although our study showed that patients are unlikely by then to have reached a plateau in their recovery. At the later follow-up, the authors reported "a trend toward better scores" in patient reported outcomes for the operative group, but this was not statistically significant.

The Canadian trial did not find an overall benefit from surgery, but was criticised for its methodology. The Swedish trial also did not find a benefit from surgery, but the authors suggested that this was only because it was underpowered. Our trial has neither of these limitations: it had a robust design and was large enough to detect a clinically significant effect. We have clearly shown that surgery is ineffective.

\section{Conclusions}

Outcomes after open reduction and internal fixation of typical, closed, displaced intra-articular fractures of the calcaneus were no better than after non-operative care at two years. We also showed that complications and reoperations were much more common in the operatively treated group. There is no justification to continue with this surgery for closed displaced intra-articular calcaneal fractures without severe displacement. Patients and their surgeons can choose safer but equally effective non-operative care.

For a clinical problem that has been beset with uncertainty for half a century, we believe that this study now provides clear 
evidence to guide practice. Future developments in surgical techniques to treat these fractures should be tested in randomised controlled trials before they are widely used.

We thank Bernard Meggitt and David Johns who stimulated our interest in calcaneal fractures; Sarah Meredith, Richard Lilford, Richard Buckley, and Roger Atkins who influenced the design of the trial; the scientific committees of the British Orthopaedic Foot and Ankle Society and the British Trauma Society who supported the trial; and the participants who agreed to take part.

UK HeFT investigators

Clinicians: Andrew Robinson (Addenbrooke's University Hospital Cambridge: ADH), Mike Hennessy, Simon Platt (Arrowe Park Hospital: APH), Clifford Butcher (Aintree University Hospital: AUH), James Ramos (Birmingham Heartlands Hospital: BHH), Declan O'Doherty, Sandeep Hemmadi (University Hospital Wales: CDF), Rick Brown (Cheltenham General Hospital: CGH), Matthew Henderson (Gloucester Royal Hospital: GRH), Tony Hui (James Cook University Hospital: JCH), Mark Phillips (King's College Hospital: KCH), Peter Giannoudis (Leeds General Infirmary: LGI), Grahame Taylor (Leicester Royal Infirmary: LRI), Andrew Kelly (Musgrove Park Hospital Taunton: MPH), Chris Blundell, Mark Davies (Northern General Hospital Sheffield: NGH), AD Patel (Norfolk and Norwich University Hospital: NNH), Alistair Henderson (Royal Bolton Hospital: RBH), lan Sharpe (Royal Devon and Exeter Hospital: RDE), Chris Walker (Royal Liverpool Hospital: RLH), John Barr (Royal Victoria Hospital: RVH), Andrew Adair (Ulster Hospital Belfast: ULS), Julian Cooper (Selly Oak Hospital Birmingham: SOH), Matthew Costa, Martin Blakemore (University Hospital Coventry and Warwickshire: UHCW), Patrick Laing, Nilesh Makwana (Wrexham Maelor Hospital: WMH)

Research associates: Troy Douglin (outcome measurement), Lynsey Brown, Sandra Carter, Jeanette Key, Joanne Bennett, Phil Clarke (ADH), Phil Dean, Angela Plant, Kerry Graves, Guy Kussabi (APH), John Moorehead, Jan Czumaj, Marie Williams, Ruth Alnwick, Ruth Lawler (AUH), Alison Miller (Tinsley), Nathan Humphries, Tim Williams (BHH), Paul Zirker, Lars Tiessen (CDF), Elaine Willmore, Sara Shannon, Kathryn Limberick (CGH), Terry Flemons, Helen Williams (GRH), Lucy Micklewright, Birgit Hanusch, Ben Cole, Kelly Willmore (JCH), Debbie Bond, Sarah Branigan (KCH), Nik Kanakaris, Tobias Lindner, Lorna Williams, Simon Aldous (LGI), Emad Mallick, Aashish Gulati, Barry Savage, Sharon Reeves (LRI), Sue Dundh, Lyndsay Osborne, Carole Chillmaid, Matt Beebee, Jenny Pickhaver, Katrina Baker (MPH), Ellen Paling, Chris Marquis, Jon Drabble, Anna Phillips (NGH), Sarah Price, Adele Cooper, Tracey Potter, Nicola Clemmence, Claire Barrett (NNH), Anne Ibberson, Lyndsay Hamlett, Angela Martin (Shore) (RBH), Anna Reffell, Robert Durman, Donna Batten, Laura Clark (RDE), Mat Hood, Michelle Nolan, Helen Haines, Suzy Broadbent, Katy Clay (RLH), Ruth Houston, Sinead McDonald, John McAlister, Denise McAuley, Christine McMillan (RVH), Katharine Dane, Heather McShane, Rachel Toland, Paula Cole (ULS), Jane Mason, Rosalyn Cooke, Stephanie Gommersall $(\mathrm{SOH})$, Rebecca Kearney, Katie McGuinness, Catherine Richmond, Helen Rowlands, Julie Petchey, John Skidmore (UHCW), Jane Evans, Helen Golledge, Cindy Wakenshaw, Ritson Lloyd (WMH)

Trial management team: Juul Achten, research manager, Rose Jarvis and Jaclyn Brown, trial co-ordinators; Jane Stokes and Charlotte Kaye, data managers.

Trial steering committee and data monitoring committee (independent members)

David Marsh (TSC Chair), Ashley Blom, Caroline Doré, Ceri Jones, Lee Shepstone (DMC Chair), Marwan Bukhari, and Alister Hart.

Contributors: DG performed a pilot study and wrote the grant application for this trial. DG, MT, SL, and ES contributed to study design and set up of the trial. DG was the chief investigator, supported by MT and SL. $\mathrm{CH}$ was responsible for analysis and reporting of the radiological data.
YK was involved in the trial set up and performed the recruitment audit. DG and NP drafted the final report; all of the UK HeFT investigators have been involved in revising the report, and all of the authors have seen and approved the final version. DG is the guarantor for this study. Funding: UK HeFT was funded by a research grant from Arthritis Research UK (grant No 15964). UK HeFT was jointly sponsored by the University of Warwick and the University Hospitals of Coventry and Warwickshire NHS Trust.

Competing interests: All authors have completed the ICMJE uniform disclosure form at www.icmje.org/coi_disclosure.pdf (available on request from the corresponding author) and declare: no support from any organisation for the submitted work; no financial relationships with any organisations that might have an interest in the submitted work in the previous three years, and no other relationships or activities that could appear to have influenced the submitted work.

Ethical approval: This study was approved by the Oxfordshire research ethics committee A 06/Q1604/58.

Data sharing: The full dataset is available from the corresponding author at damian.griffin@warwick.ac.uk.

Transparency: The lead author affirms that this manuscript is an honest, accurate, and transparent account of the study being reported; that no important aspects of the study have been omitted; and that any discrepancies from the study as planned and registered have been explained.

1 Mitchell MJ, McKinley JC, Robinson CM. The epidemiology of calcaneal fractures. Foot (Edinb) 2009;19:197-200.

2 NHS Information Centre. Hospital episode statistics: 2000-2012. www.hesonline.nhs.uk. 3 Agency for Healthcare Research and Quality. HCUP nationwide inpatient sample. 2010. www.hcup-us.ahrq.gov/nisoverview.jsp.

4 Dhillon MS, Aggarwal S, Dhatt S, Jain M. Epidemiological pattern of foot injuries in India: preliminary assessment of data from a tertiary hospital. J Postgrad Med Edu Res 2012;46:144-7.

5 Tadros AM, Eid HO, Abu-Zidan FM. Epidemiology of foot injury in a high-income developing country. Injury 2010;41:137-40.

6 Crosby LA, Fitzgibbons T. Intraarticular calcaneal fractures. Results of closed treatment. Clin Orthop Relat Res 1993;May (290):47-54.

7 Cotton F, Henderson F. Results of fracture of the os calcis. J Bone Joint Surg Am 1916;s2-14:290-8.

8 Schepers T, van Lieshout EMM, van Ginhoven TM, Heetveld MJ, Patka P. Current concepts in the treatment of intra-articular calcaneal fractures: results of a nationwide survey. Int Orthop 2008:32:711-5.

9 Essex-Lopresti P. The mechanism, reduction technique, and results in fractures of the os calcis. Br J Surg 1952;39:395-419.

10 Charnley J. The closed treatment of common fractures. Cambridge University Press, 2003.

11 Ibrahim T, Rowsell M, Rennie W, Brown AR, Taylor GJ, Gregg PJ. Displaced intra-articular calcaneal fractures: 15-year follow-up of a randomised controlled trial of conservative versus operative treatment. Injury 2007;38:848-55.

12 Eastwood DM, Langkamer VG, Atkins RM. Intra-articular fractures of the calcaneum. Part II: open reduction and internal fixation by the extended lateral transcalcaneal approach. J Bone Joint Surg Br 1993;75:189-95.

13 Tennent TD, Calder PR, Salisbury RD, Allen PW, Eastwood DM. The operative management of displaced intra-articular fractures of the calcaneum: a two-centre study using a defined protocol. Injury 2001;32:491-6.

14 Jain S, Jain AK, Kumar I. Outcome of open reduction and internal fixation of intraarticular calcaneal fracture fixed with locking calcaneal plate. Chin J Traumatol 2013;16:355-60.

15 Bajammal S, Tornetta P, Sanders D, Bhandari M. Displaced intra-articular calcaneal fractures. J Orthop Trauma 2005;19:360-4.

16 Gougoulias N, Khanna A, McBride DJ, Maffulli N. Management of calcaneal fractures: systematic review of randomized trials. Br Med Bull 2009;92:153-67.

17 Jiang N, Lin Q-R, Diao X-C, Wu L, Yu B. Surgical versus nonsurgical treatment of displaced intra-articular calcaneal fracture: a meta-analysis of current evidence base. Int Orthop 2012;36:1615-22.

18 Bruce J, Sutherland A. Surgical versus conservative interventions for displaced intra-articular calcaneal fractures. Cochrane Database Syst Rev 2013;1:CD008628.

19 Folk JW, Starr AJ, Early JS. Early wound complications of operative treatment of calcaneus fractures: analysis of 190 fractures. J Orthop Trauma 1999;13:369-72.

20 Sanders R, Fortin P, DiPasquale T, Walling A. Operative treatment in 120 displaced intraarticular calcaneal fractures. Results using a prognostic computed tomography scan classification. Clin Orthop Relat Res 1993;May(290):87-95.

21 Epstein N, Chandran S, Chou L. Current concepts review: intra-articular fractures of the calcaneus. Foot Ankle Int 2012:33:79-86.

22 Kerr PS, Prothero DL, Atkins RM. Assessing outcome following calcaneal fracture: a rational scoring system. Injury 1996;27:35-8.

23 Pozo JL, Kirwan EO, Jackson AM. The long-term results of conservative management of severely displaced fractures of the calcaneus. J Bone Joint Surg Br 1984;66:386-90.

24 Brooks R. EuroQol: the current state of play. Health Policy 1996;37:53-72.

25 Ware JE, Sherbourne CD. The MOS 36 -item short-form health survey (SF-36). I. Conceptual framework and item selection. Med Care 1992;30:473-83. 


\section{What is already known on this topic}

Displaced intra-articular fractures of the calcaneus (heelbone) are a serious injury, often caused by falls, and most common in manual workers and labourers

Outcomes are often poor, with many patients disabled and unable to return to previous work

For years there has been controversy about whether surgery to reconstruct the acute fracture is safe and can improve outcomes, but evidence from randomised controlled trials has been insufficient to guide practice

\section{What this study adds}

Surgery by open reduction and internal fixation in patients with typical displaced calcaneal fractures does not improve outcomes compared with non-operative care

Operative treatment was associated with a higher rate of complications

26 Kitaoka HB, Alexander IJ, Adelaar RS, Nunley JA, Myerson MS, Sanders M. Clinical rating systems for the ankle-hindfoot midfoot hallux and lesser toes. Foot Ankle Int 1994;15:349-53.

27 Menadue C, Raymond J, Kilbreath SL, Refshauge KM, Adams R. Reliability of two goniometric methods of measuring active inversion and eversion range of motion at the ankle. BMC Musculoskelet Disord 2006:7:60.

28 Thornes BS, Collins AL, Timlin M, Corrigan J. Outcome of calcaneal fractures treated operatively and non-operatively. the effect of litigation on outcomes. Ir J Med Sci 2002;171:155-7.

29 Buckley R, Tough S, McCormack R, Pate G, Leighton R, Petrie D, et al. Operative compared with nonoperative treatment of displaced intra-articular calcaneal fractures: a prospective randomized controlled multicenter trial. J Bone Joint Surg Am 2002;84-A:1733-44

30 Brookes ST, Whitley E, Peters TJ, Mulheran PA, Egger M, Davey Smith G. Subgroup analyses in randomised controlled trials: quantifying the risks of false-positives and false-negatives. Health Technol Assess 2001;5:1-56.

31 Robinson RO, Herzog W, Nigg BM. Use of force platform variables to quantify the effects of chiropractic manipulation on gait symmetry. J Manipulative Physiol Ther 1987:10:172-6. 32 Jenkinson C, Stewart-Brown S, Petersen S, Paice C. Assessment of the SF-36 version 2 in the United Kingdom. J Epidemiol Community Health 1999;53:46-50.
33 Holecek T, Dedek T. Open versus closed method of operative treatment of calcaneal fractures. Eur J Trauma 2002;28(Supp 1):84.

34 O'Farrell DA, O'Byrne JM, McCabe JP, Stephens MM. Fractures of the os calcis: improved results with internal fixation. Injury 1993;24:263-5.

35 Thordarson DB, Krieger LE. Operative vs. nonoperative treatment of intra-articular fractures of the calcaneus: a prospective randomized trial. Foot Ankle Int 1996:17:2-9.

36 Zelen M. A new design for randomized clinical trials. N Engl J Med 1979;300:1242-5.

37 Agren P-H, Wretenberg P, Sayed-Noor AS. Operative versus nonoperative treatment of displaced intra-articular calcaneal fractures: a prospective randomized controlled multicenter trial. J Bone Joint Surg Am 2013;95:1351-7.

Accepted: 25 June 2014

\section{Cite this as: BMJ 2014;349:g4483}

This is an Open Access article distributed in accordance with the Creative Commons Attribution Non Commercial (CC BY-NC 3.0) license, which permits others to distribute, remix, adapt, build upon this work non-commercially, and license their derivative works on different terms, provided the original work is properly cited and the use is non-commercial. See: http://creativecommons.org/licenses/by-nc/3.0/. 


\section{Tables}

Table 1| Baseline characteristics of 151 trial participants. Values are either counts (percentages) or means (standard deviations)

\begin{tabular}{lcc} 
Characteristics & Operative $(\mathbf{n}=\mathbf{7 3})$ & Non-operative $(\mathbf{n}=\mathbf{7 8})$ \\
Males $(\%)$ & $64(88)$ & $63(81)$ \\
\hline Age (years) & $44.8(14.8)$ & $48.2(16.5)$ \\
\hline Body mass index & $25.4(5.1)$ & $25.3(3.9)$ \\
\hline Smokers (\%) & $37(51)$ & $44(56)$ \\
\hline Diabetes (\%) & $1(1)$ & $3(4)^{\star}$ \\
\hline Kerr-Atkins & $99(1)$ & $99(4)$ \\
\hline Sanders classification $(\%)^{*}:$ & & $35(46)$ \\
\hline 2 & $35(49)$ & $30(39)$ \\
\hline 3 & $27(38)$ & $11(15)$ \\
\hline 4 & $9(13)$ & $53(9)$ \\
\hline SF-36 PCS & $51(10)$ & $56(10)$ \\
\hline SF-36 MCS & $55(10)$ & $0.9(0.2)$
\end{tabular}

*One participant with unknown diabetes status; Sanders classification from 147 participants (four could not be classified precisely). 
Table 2| Means and standard deviations of outcomes at two years follow-up, and estimated treatment effects after adjustment. Values are means (standard deviations) unless stated otherwise

\begin{tabular}{|c|c|c|c|c|c|c|c|}
\hline \multirow[b]{2}{*}{ Outcomes } & \multicolumn{2}{|c|}{ Operative } & \multicolumn{2}{|c|}{ Non-operative } & \multicolumn{2}{|c|}{ Difference $(95 \% \mathrm{Cl})$} & \multirow[b]{2}{*}{$P$ value } \\
\hline & No & Values & No & Values & Raw & Adjusted $^{*}$ & \\
\hline \multicolumn{8}{|c|}{ Primary outcome (Kerr-Atkins score) } \\
\hline All participants & 69 & $69.8(21.8)$ & 74 & $65.7(23.7)$ & 4.1 & $0.0(-7.1$ to 7.0$)$ & 0.993 \\
\hline \multicolumn{8}{|l|}{ Subgroups: } \\
\hline Sanders 2 & 33 & $74.3(20.4)$ & 34 & $70.3(24.9)$ & 4.0 & $1.0(-8.9$ to 10.9$)$ & 0.842 \\
\hline Sanders 3 or 4 & 34 & $66.1(21.9)$ & 38 & $63.0(22.2)$ & 3.3 & $3.1(-7.6$ to 13.8$)$ & 0.571 \\
\hline Males & 60 & $71.9(20.1)$ & 59 & $67.7(23.5)$ & 4.2 & $1.4(-5.7$ to 8.5$)$ & 0.706 \\
\hline Females & 9 & $55.7(28.2)$ & 15 & $57.5(23.8)$ & -1.8 & $0.2(-20.6$ to 21.0$)$ & 0.987 \\
\hline \multicolumn{8}{|l|}{ Secondary outcomes } \\
\hline \multicolumn{8}{|l|}{$\begin{array}{l}\text { Patient reported } \\
\text { outcomes: }\end{array}$} \\
\hline SF-36 PCS & 54 & $43.7(11.1)$ & 62 & $37.0(13.1)$ & 6.7 & $3.3(-0.9$ to 7.6$)$ & 0.131 \\
\hline SF-36 MCS $†$ & 54 & $53.4(11.4)$ & 62 & $53.6(12.3)$ & -0.2 & $-2.5(-6.7$ to 1.6$)$ & 0.235 \\
\hline EQ-5D & 59 & $0.72(0.22)$ & 62 & $0.66(0.27)$ & 0.07 & $0.03(-0.06$ to 0.11$)$ & 0.568 \\
\hline AOFAS & 54 & $79.2(16.2)$ & 60 & $76.8(19.7)$ & 2.5 & $0.1(-6.5$ to 6.7$)$ & 0.976 \\
\hline Return to work (\%) & 45 & 84.4 & 36 & 88.9 & $\S 0.68$ & $\S 0.64$ (0.12 to 3.32$)$ & 0.599 \\
\hline \multicolumn{8}{|l|}{ Clinical measurements: } \\
\hline Dorsiflexion (\%)‡ & 49 & $-35.3(115.1)$ & 54 & $-28.1(67.8)$ & -7.2 & $-17.0(-54.7$ to 20.8$)$ & 0.381 \\
\hline Plantarflexion (\%) & 49 & $-25.3(37.3)$ & 55 & $-27.7(41.7)$ & 2.4 & $-5.9(-22.9$ to 11.0$)$ & 0.493 \\
\hline Inversion (\%) & 48 & $-66.4(47.8)$ & 54 & $-56.3(55.4)$ & -10.1 & $-18.2(-39.0$ to 2.6$)$ & 0.090 \\
\hline Eversion (\%) & 48 & $-71.7(57.9)$ & 54 & $-56.4(52.7)$ & -15.3 & $-13.2(-37.4$ to 10.9$)$ & 0.287 \\
\hline Heel width (mm) & 48 & $67.9(7.7)$ & 54 & $68.0(7.0)$ & -0.1 & $-0.9(-3.9$ to 2.2$)$ & 0.571 \\
\hline Walking speed $(\mathrm{m} / \mathrm{s})$ & 54 & $1.19(0.24)$ & 58 & $1.05(0.29)$ & 0.15 & $0.05(-0.02$ to 0.17$)$ & 0.137 \\
\hline \multicolumn{8}{|l|}{ Gait symmetry indices: } \\
\hline Contact duration (\%) $\ddagger$ & 45 & $-0.9(7.4)$ & 50 & $1.1(8.2)$ & -2.0 & $-3.1(-6.3$ to 0.2$)$ & 0.072 \\
\hline Midfoot PP (\%) & 45 & $24.7(76.5)$ & 51 & $27.3(72.3)$ & -2.6 & $-6.7(-39.9$ to 26.5$)$ & 0.693 \\
\hline 1st metatarsal PP (\%) & 45 & $-31.9(64.7)$ & 51 & $-9.9(72.8)$ & -22.0 & $-21.8(-50.7$ to 7.1$)$ & 0.144 \\
\hline 5th metatarsal PP (\%) & 45 & $11.2(68.6)$ & 51 & $3.0(61.4)$ & 8.2 & $4.7(-22.3$ to 31.7$)$ & 0.735 \\
\hline Force time integral (\%) & 45 & $-9.2(20.8)$ & 51 & $-9.3(30.1)$ & 0.1 & $-0.8(-11.9$ to 10.4$)$ & 0.895 \\
\hline
\end{tabular}

$\mathrm{PP}=$ peak pressure.

*Mixed effects regression with baseline score, age, sex, smoking status, employment status, Sanders classification, and compensation status fitted as covariates (fixed effects) and recruiting centre as random effect.

†SF-36 PCS (physical component) and SF-36 MCS (mental component) normative scores: mean 50 (SD 10)

$\ddagger \%$ difference relative to non-injured foot-that is, $100 \times$ (injured-non-injured)/0.5×(injured+non-injured), for unilateral fractures only.

§Odds ratio for binary outcome. 
Table 3| Details and rates of complications and reoperations by treatment group

\begin{tabular}{|c|c|c|}
\hline Reported complications and reoperations & Operative $(n=73)$ & Non-operative $(n=78)$ \\
\hline \multicolumn{3}{|l|}{6 weeks* } \\
\hline \multicolumn{3}{|l|}{ Treatment of surgical site infections: } \\
\hline Antibiotics and dressings & 6 & 0 \\
\hline Wound debridement & 2 & 0 \\
\hline Removal of screws/plates & 2 & 0 \\
\hline \multicolumn{3}{|l|}{6 monthst } \\
\hline \multicolumn{3}{|l|}{ Treatment of surgical site infections: } \\
\hline Antibiotics and dressings & 3 & 0 \\
\hline Removal of screws/plates & 1 & 0 \\
\hline Removal of prominent metalwork & 1 & 0 \\
\hline Sural nerve injury & 0 & 1 \\
\hline \multicolumn{3}{|l|}{12 months } \\
\hline None & 0 & 0 \\
\hline \multicolumn{3}{|l|}{24 monthsł } \\
\hline Removal of prominent metalwork & 2 & 0 \\
\hline Subtalar fusion for chronic pain§ & 0 & 3 \\
\hline \multicolumn{3}{|l|}{ Reported by participants (No (\%)): } \\
\hline Neurovascular injury & $0(0)$ & $1(1)$ \\
\hline Surgical site infection & $14(19)$ & $0(0)$ \\
\hline Reoperations & $8(11)$ & $3(4)$ \\
\hline Any complication or reoperation $\mathbb{1}$ & $17(23)$ & $3(4)$ \\
\hline
\end{tabular}

${ }^{*}$ Reported from nine centres.

†Complications in operative group reported from two centres, complication in non-operative group from another centre.

$\ddagger$ Complications in operative group reported from one centre, complications in non-operative group reported from three centres.

§Includes participant with sural nerve injury.

ףSome participants experienced more than one event. 


\section{Figures}

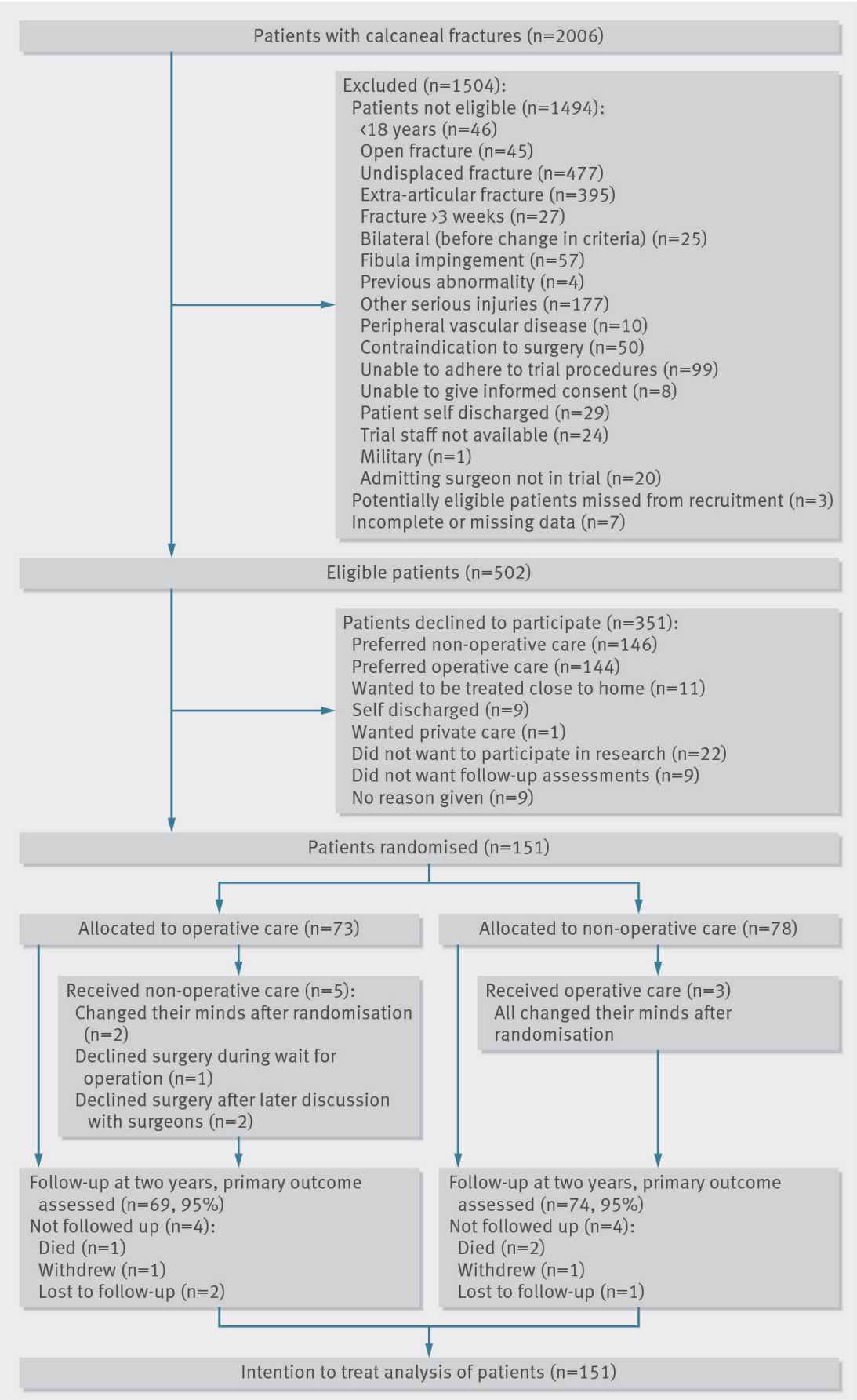

Fig 1 Trial profile 

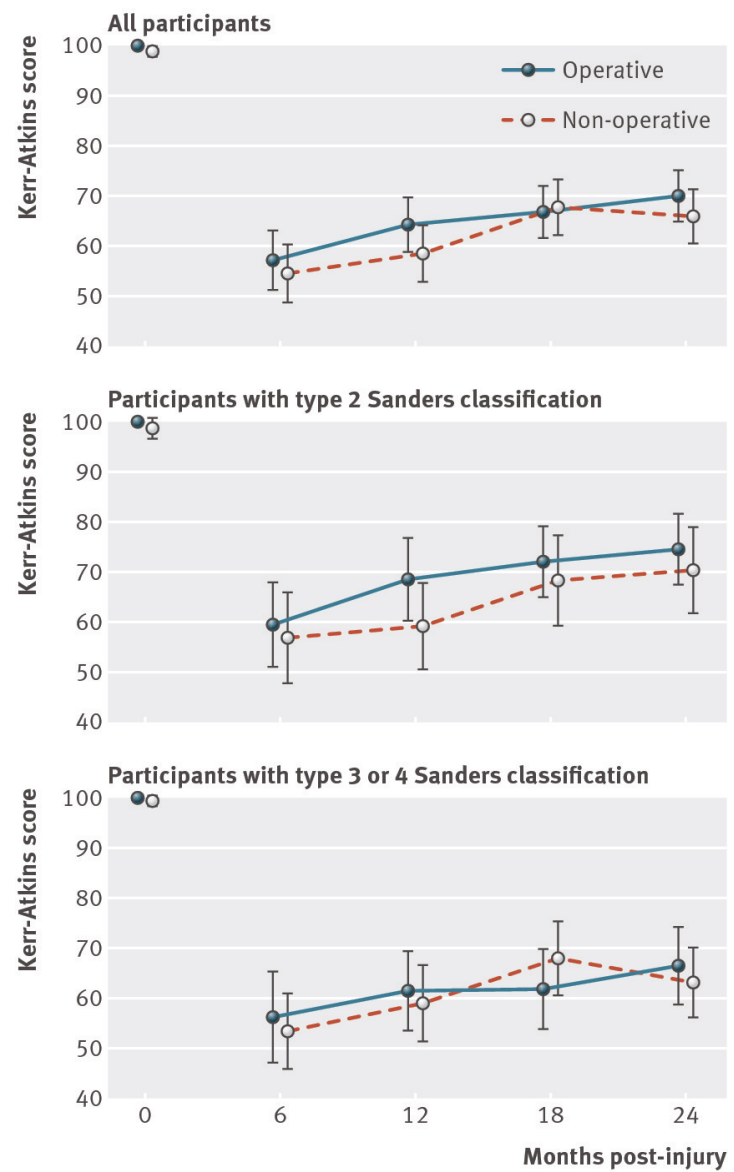

Fig 2 Kerr-Atkins scores for calcaneal fracture and 95\% confidence intervals at baseline (before injury) and 6, 12, 18, and 24 months after injury for all participants and for those with type 2 and with type 3 or 4 fractures (Sanders classification)

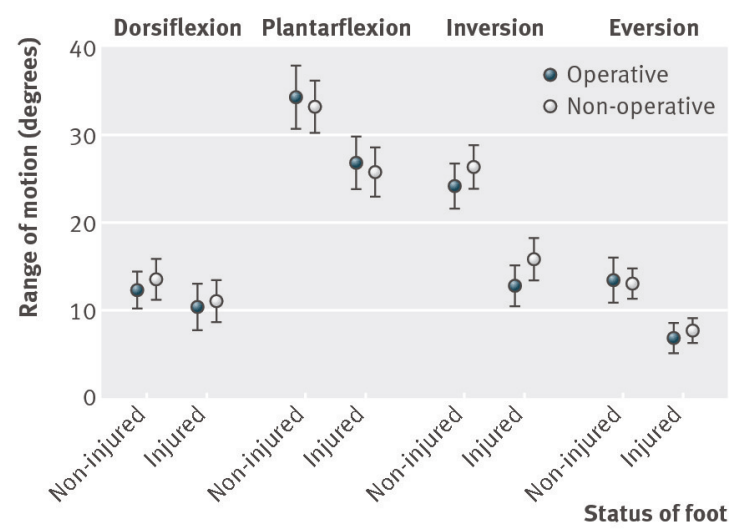

Fig 3 Range of motion in degrees (group means and 95\% confidence intervals) and gait symmetry data at 24 months post-injury for injured and non-injured feet 


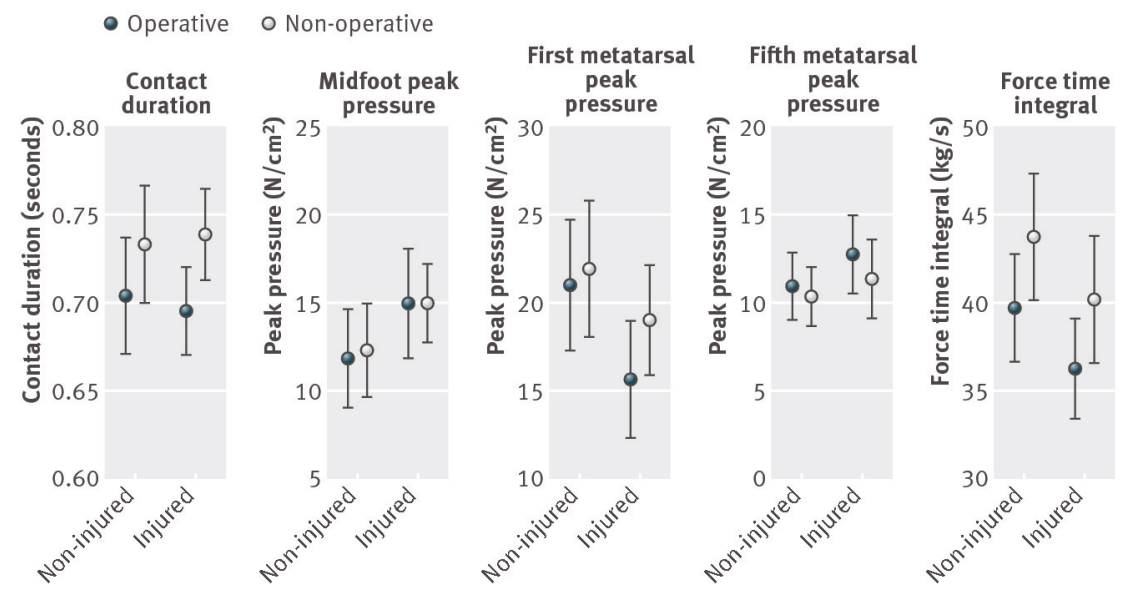

Fig 4 Gait metrics (group means and 95\% confidence intervals) at 24 months post-injury for injured and non-injured feet 